\title{
A Review on Corticosteroids and Its Clinical Complications on Long Term Exposure
}

\author{
Lakshmi Surekha $K^{1}$, Naveen Reddy $P^{2}$, Rasajna $G^{3}$ and \\ Bhavani B ${ }^{4 *}$ \\ ${ }^{1,2,3}$ Koringa College of Pharmacy \\ Korangi, East Godavari, AP, India \\ 4* Professor \& Principal, Koringa College of Pharmacy, \\ Korangi, East Godavari, AP, India
}

\begin{abstract}
Corticosteroids and their biologically active synthethic derivatives differ in their metabolic and electrolyte-regulating activities. These agents are employed at physiological doses for replacement therapy when endogenous product is impaired. While corticosteroids are relatively inexpensive \& commonly used as treatment for a variety of conditions, long term use is known to be associated with certain toxicities. Increasing application of steroid therapy during operative period for various purposes. The decision for corticosteriodal therapy always requires careful consideration of the relative risks \& benefits in each patient. Although steroids may have a lifesaving activity on general diseases, their dosage should be reviewed regularly by prescribers to reduce the subsequent complications of long-term therapy.
\end{abstract}

Keywords: Corticosteroids, Adverse effects, Long term exposure

\section{Introduction}

corticosteroids have become one of the most widely used from 1940 onwards and are effective treatments for various inflammatory and autoimmune disorders. They are used as replacement therapy in adrenal insufficiency (at physiologic doses) as well as in supraphysiologic doses for the management of various dermatologic, ophthalmologic, rheumatologic, pulmonary, hematologic, and gastrointestinal (GI) disorders. In the field of respirology, systemic corticosteroids are used for the treatment of acute exacerbations of chronic obstructive pulmonary disease (COPD) and severe, uncontrolled asthma, as well as for inflammatory parenchymal lung diseases such as hypersensitivity pneumonitis and immune mediated vasculitis $^{(1)}$. Corticosteroids and their biologically active synthetic derivatives will differ in their metabolic (glucocorticoid) and electrolyteregulating (mineralocorticoid) activities. These agents are employed at physiological doses for replacement therapy when endogenous production is impaired. In addition, glucocorticoids potently suppress inflammation, and their use in a variety of inflammatory and autoimmune diseases makes them among the most frequently prescribed classes of drugs ${ }^{(2,3)}$. However, despite the potentially beneficial clinical effects of these agents, such use is also associated with serious risks, especially at high doses for extended periods ${ }^{(1)}$ Well-known adverse events (AEs) associated with systemic corticosteroid use include osteoporosis, cardiovascular disease, impaired immune response and wound healing, alterations in glucose and lipid metabolism, and psychiatric disturbances ${ }^{(1,4)}$ they are sometimes termed as the "double-edged sword" in the field of medicine. Such complications may decrease a patient's quality of life and can also be costly to manage ${ }^{(5,6)}$

\section{Regulation of Cortisol Secretion}

The following three major mechanisms control ACTH release and the Cortisol secretion.

(a) Negative feedback mechanism: the most important stimulus for secretion of cortisol is the release of ACTH from anterior pituitary. The secretion of ACTH in anterior pituitary is determined by two hypothalamic neurohormones (diurnal release of CRF and AVP) that act synergistically.Circulating cortisol also exerts a direct negative feedback on the hypothalamus and 
anterior pituitary to decrease the release of CRF and ACTH from respective sites ${ }^{(7,8,9)}$.

(b) Diurnal variation: cortisol is secreted from adrenal gland in an episodic manner and frequency of pulses follows a circadian rhythm that is dependent on both day-night and sleep-wake patterns and is disrupted by alternating day-night shift working patterns and by long distance travel across time zones. It may take up to 2 weeks for circadian rhythm to reset to an altered day-night cycle. Levels are the highest in the morning on waking and the lowest in the middle of evening. Maximal cortisol level occurs at 4-6 am and is lower at $4-8 \mathrm{pm}^{(7,8,9)}$

(c) Stress: Stress such as physical (trauma, surgery, exercise); psychological (pain, anxiety, apprehension); physiological (nausea, fever and hypoglycemia) can over ride the negative feedback mechanism and diurnal variation.Cortisol rises immediately (within minutes) and dramatically during stress. This elevation protects key metabolic functions and puts an important "brake" on potentially damaging inflammatory responses to infection and injury ${ }^{(7)}$.

\section{Adverse Effects Associated with long term use Of Corticosteroids}

As discussed above corticosteroids are double edged sword, prolonged use of steroids mainly systemic steroids leads to adverse effects like osteoporosis and fractures; HPA-axis suppression; Cushing syndrome and weight gain; hyperglycaemia/ diabetes; CVD and dyslipidaemia;myopathy; cataracts and glaucoma; psychiatric disturbances; immunosuppression; as well as other GI and dermatologic events ${ }^{(1)}$.Mechanisms of adverse effects during the administration of Glucocorticoids observed chronic disease (eg, prednisone or prednisolone) do not have significant mineralocorticoid, androgenic, or estrogenic activity; thus, their major adverse effects result from inhibition of hypothalamic-pituitaryadrenal function and the development of iatrogenic Cushing's syndrome. The effects of glucocorticoids are mediated by differences in bioavailability, receptor activation by phosphorylation, translocation, and repression/activation of gene expression. This leaves multiple sites where differences among glucocorticoids and/or differences among individuals can result in differences in efficacy and toxicity. It helps explain the differential resistance to therapy and toxicities among patients ${ }^{(10,11)}$. Below figure1 is the over view of long term effects of steroids.

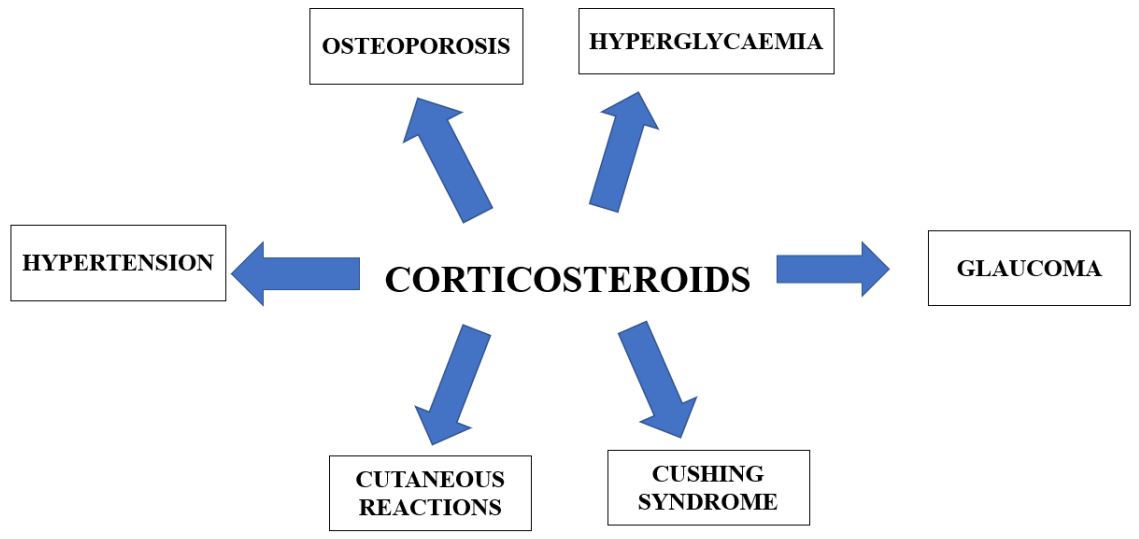

Fig 1: Effects of long term use of corticosteroids.

\section{OSTEOPOROSIS}

Corticosteroids affect calcium and bone metabolism in many ways:Steroids increase the natural rate of bone breakdown, Steroids decrease bone formation, Steroids decrease the amount of calcium absorbed by the intestine, Steroids increase calcium excretion through the kidneys, Steroids work directly on target tissues in bone to increase resorption and decrease formation ${ }^{(12)}$.Corticosteroids stimulate osteoclastic activity initially (first 6-12 months of therapy), followed by a decrease in bone formation by suppressing osteoblastic activity in the bone marrow, decreasing osteoblast function and life span, and promoting the apoptosis of osteoblasts and osteocytes ${ }^{(13,14)}$.

\section{HYPERGLYCAEMIA}

The effect of glucocorticoids on glucose metabolism is likely the result of impairment of multiple pathways including beta cell dysfunction 
(sensitivity to glucose and ability to release insulin) and insulin resistance in other tissue. The role of beta cell function and other tissues' sensitivity to insulin may be different depending on whether the glucocorticoid effect is acute or chronic. prolonged glucocorticoid exposure showed partial recovery of beta cell function but similarly impaired glucose tolerance, suggesting additional factors are important in SIDM other than beta cell dysfunction ${ }^{(15,16)}$.

\section{GLAUCOMA}

Steroid-induced glaucoma is a form of secondary open-angle glaucoma occurring as an adverse effect of corticosteroid therapy. It is usually associated with topical steroid use, but it may develop with oral, intravenous, inhaled, and periocular steroid administration by causing a decrease in aqueous outflow facility. The association of steroids and glaucoma was first established in 1950 when systemic administration of the adrenocorticotropin hormone was shown to increase intraocular pressure (IOP) ${ }^{(17)}$.

\section{CUSHING SYNDROME}

Cushing's syndrome is an endocrine disorder caused when the adrenal glands produce excess cortisol. Also known as hypercortisolism, Cushing's syndrome can occur for various reasons. One of the most common causes is long-term exposure to corticosteroid medications like prednisone. Cushing's syndrome that's caused by steroids is called iatrogenic (or exogenous) Cushing's syndrome ${ }^{(18)}$. long-term exposure to cortisol either from natural production or corticosteroid medication use can result in increased glucose levels, insulin resistance, and weight gain $^{(19)}$. Prolonged corticosteroid therapy commonly causes weight gain and redistribution of adipose tissue that result in Cushingoid features (truncal obesity, facial adipose tissue [i.e., moon face], and dorsocervical adipose tissue $)^{(20)}$.

\section{CUTANEOUS REACTIONS}

Corticosteroids induce atrophic changes in the skin that can lead to skin thinning and fragility, purpura and red striae. Skin thinning and purpura are usually reversible upon discontinuation of therapy, but striae are permanent. Purpura generally affect the sun exposed areas of the dorsum of the hands and forearms, as well as the sides of the neck, face, and lower legs, and are usually not accompanied by palpable swelling ${ }^{(1,21,22)}$.

\section{HYPERTENSION}

corticosteroid administration is a risk factor for cardiovascular disease, Knowledge of the method of action of steroids has grown rapidly over the last few years, providing greater understanding of both beneficial and unwanted effects. Some of these actions might be seen as harmful to heart and circulation. Hypertension was one of the features of Cushing's original report of endogenous hypercortisolism $^{(23)}$ and there is still widespread acceptance that high blood pressure is a sequel of glucocorticoid excess from whatever cause ${ }^{(24)}$. Several studies have suggested that long-term lowdose corticosteroid therapy can cause hypertension $^{(25,26)}$. found a highly significant rise in systolic blood pressure (135.9 to 147) in 195 patients with either asthma or RA treated for at least one year with prednisone or prednisolone compared to those not on steroids ${ }^{(28)}$. Below figure 2 represents the overview of mechanism of effects of long term use of steroids on the individuals.

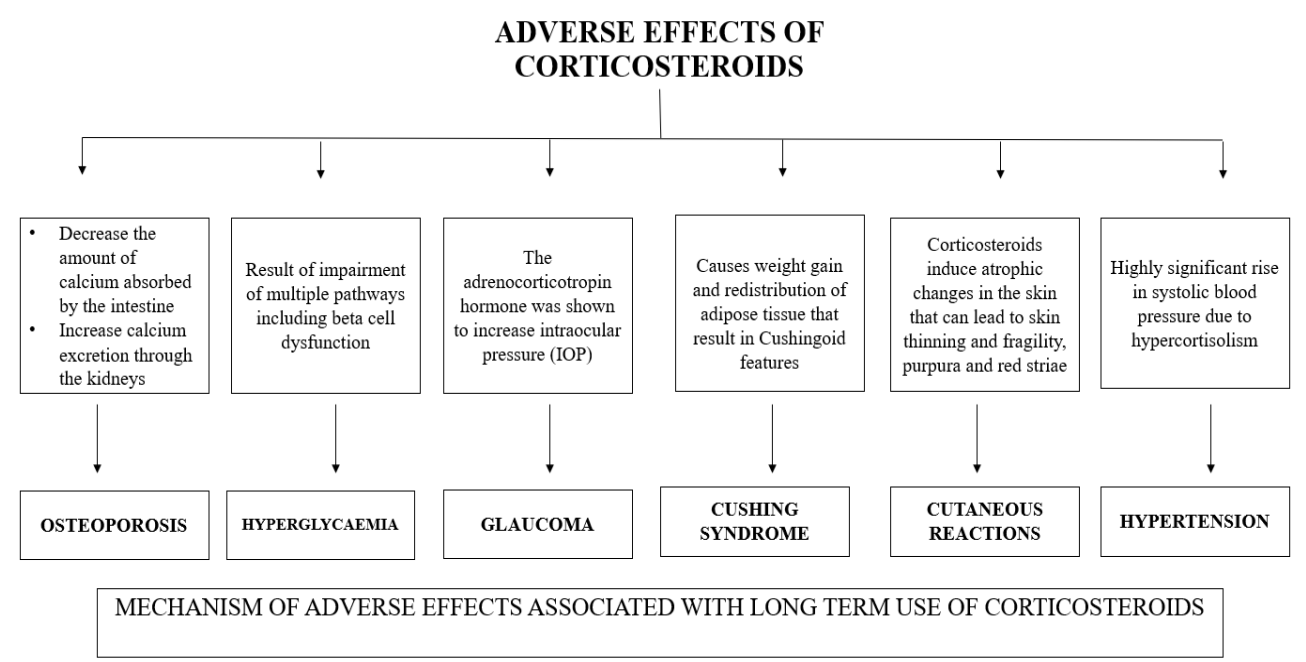

Fig :2 Mechanism of effects of corticosteroids in long term use 


\section{CONCLUSION}

In view of different adverse effects caused by corticosteroids, it is surprising that a close relationship between corticosteroid usage and osteoporosis, hyperglycaemia, glaucoma, cutaneous reactions cardiovascular damage has not been more thoroughly investigated. A considerable evidence is that body that has been presented with at the very least, casts suspicious about the effects of the drugs that are currently in widespread use. The lack of attention previously given to this subject probably reflects the fact that therapy to the patients which is usually given to those of people effected with asthma, RA and other autoimmune diseases for prolonged period of time is leading to these complications.Because of these complications a small but genuine effect may be masked.

It is a hope that, in the future, safer and less toxic steroid-sparing agents will be available. Although steroids may have a lifesaving activity on general diseases, their dosage should be reviewed regularly by prescribers to reduce the subsequent complications of long-term therapy.

\section{References}

[1] Liu D, Ahmet A, Ward L, et al. A practical guide to the monitoring and management of the complications of systemic corticosteroid therapy. Allergy Asthma Clin Immunol. 2013;9:1-25.

[2] Bruton L L., Lazo J S, and Parker K L, Goodman \& Gilman'S the Pharmacological Basis of Therapeutics, 11th edition, 2006.

[3] Text book of Human Anatomy by Gray's, 68th edition

[4] Wan ES, Qiu W, Baccarelli A, et al. Systemic steroid exposure is associated with differential methylation in chronic obstructive pulmonary disease. Am J Respir Crit Care Med. 2012;186:1248-1255.

[5] Manson SC, Brown RE, Cerulli A, et al. The cumulative burden of oral corticosteroid side effects and the economic implications of steroid use. Respir Med. 2009;103: 975-994

[6] Shah M, Chaudhari S, McLaughlin T, et al. Cumulative burden of oral corticosteroid adverse effects and the economic implications of corticosteroid use in patients with systemic lupus erythematosus. Clin Ther. 2013;35: 486-497.

[7] C. Jung and W. J. Inder, "Management of adrenal insufficiency during the stress of medical illness and surgery," Medical Journal of Australia, vol. 188, no. 7, pp. 409-413, 2008

[8] Braunwald et al., Harrison's Principles of Internal Medicine by Kasper, 17th edition
[9] Review of Medical Physiology by William F. Ganong, 24th edition.

[10] Schäcke H, Döcke WD, Asadullah K. Mechanisms involved in the side effects of glucocorticoids. Pharmacol Ther 2002; 96:23.

[11] Ramamoorthy S, Cidlowski JA. Exploring the molecular mechanisms of glucocorticoid receptor action from sensitivity to resistance. Endocr Dev 2013; 24:41.

[12] https://www.verywellhealth.com/what-toknow-about-corticosteroid-inducedosteoporosis-190176

[13] Weinstein RS, Jilka RL, Parfitt AM, Manolagas SC: Inhibition of osteoblastogenesis and promotion of apoptosis of osteoblasts and osteocytes by glucocorticoids. Potential mechanisms of their deleterious effects on bone. J Clin Invest 1998, 102:274-282

[14] Yao W, Cheng Z, Busse C, Pham A, Nakamura MC, Lane NE: Glucocorticoid excess in mice results in early activation of osteoclastogenesis and adipogenesis and prolonged suppression of osteogenesis: a longitudinal study of gene expression in bone tissue from glucocorticoid-treated mice. Arthritis Rheum 2008, 58:1674-1686.

[15] van Raalte DH, Nofrate V, Bunck MC, et al. Acute and 2-week exposure to prednisolone impair different aspects of beta-cell function in healthy men. Eur J Endocrinol. 2010;162:729-735.

[16] Jessica L. Hwang et.al, Steroid-induced diabetes: a clinical and molecular approach to understanding and treatment, Diabetes Metab Res Rev. 2014 Feb; 30(2): 96-102.

[17] McLean JM. Use of ACTH and cortisone. Trans Am Ophthalmol Soc. 1950;48:293-296.

[18] Nieman LK. Diagnosis of Cushing's Syndrome in the Modern Era. Endocrinol Metab Clin North Am. 2018;47(2):259-273. doi:10.1016/j.ecl.2018.02.001

[19] Geer EB, Islam J, Buettner C. Mechanisms of glucocorticoid-induced insulin resistance: focus on adipose tissue function and lipid metabolism. Endocrinol Metab Clin North Am. doi:10.1016/j.ecl.2013.10.005

[20] Curtis JR, Westfall AO, Allison J, Bijlsma JW, Freeman A, George V, Kovac SH, Spettell CM, Saag KG: Population-based assessment of adverse events associated with long-term glucocorticoid use. Arthritis Rheum 2006, 55:420-426.

[21] Haimovici R, Gragoudas ES, Duker JS, Sjaarda RN, Eliott D: Central serous chorioretinopathy associated with inhaled or 
intranasal corticosteroids. Ophthalmol 1997, 104:1653-1660.

[22] Schellenberg R, Adachi JDR, Bowie D, Brown J, Guenther L, Kader T, Trope GE: Oral corticosteroids in asthma: a review of benefits and risks. Can Respir J 2007, 14(Suppl C):1C-7C.

[23] Cushing, H. The basophil adenomas of the putuitary body and their clinical manifestations (pituitary basophilism). Bull Johns Hopkins Hosp 1932, 50: 137

[24] Krakoff, L.R. Glucocorticoid excess syndromes causing hypertension. Cardiol Clin 1988, 6: 537-545.

[25] Savage, O., Copeman, W.S.C., Chapman, L., Weils, M.V. \& Treadwell, B.L.J. Pituitary and adrenal hormones in rheumatoid arthritis. Lancet 1962, 1: 232-235.

[26] Treadwell, B.L.J., Sever, E.D., Savage, 0. \& Copeman, W.S.C. Side-effects of long-term treatment with corticosteroids and corticotrophin. Lancet 1964, i: 1121.

[27] Smyllie, H.C. \& Connolly, C.K. Incidence of serious complications of corticosteroid therapy in respiratory disease. Thorax 1968, 23: 571581.

[28] Jackson, S.H.D., Beevers, D.G. \& Myers, K. Does longterm low-dose corticosteriod therapy cause hypertension? Clin Sci 1981, 61: 381S-383S. 